Tuning growth direction of catalyst-free $\ln \mathrm{As}(\mathrm{Sb})$ nanowires with indium droplets

This content has been downloaded from IOPscience. Please scroll down to see the full text.

2017 Nanotechnology 28054001

(http://iopscience.iop.org/0957-4484/28/5/054001)

View the table of contents for this issue, or go to the journal homepage for more

Download details:

IP Address: 128.178.120.45

This content was downloaded on 30/04/2017 at 14:36

Please note that terms and conditions apply.

You may also be interested in:

Foreign-catalyst-free growth of $\operatorname{InAs} / \mathrm{InSb}$ axial heterostructure nanowires on $\mathrm{Si}$ (111) by molecular-beam epitaxy

Hyok So, Dong Pan, Lixia Li et al.

Catalyst-free growth of InAs nanowires on $\mathrm{Si}$ (111) by CBE

U P Gomes, D Ercolani, N V Sibirev et al.

MBE growth of self-assisted InAs nanowires on graphene

Jung-Hyun Kang, Yuval Ronen, Yonatan Cohen et al.

Palladium assisted hetroepitaxial growth of an InAs nanowire by molecular beam epitaxy

R Perumal, Zhixin Cui, Patniarche Gille et al.

Faceting, composition and crystal phase evolution in III-V antimonide nanowire heterostructures revealed by combining microscopy techniques

Tao Xu, Kimberly

A Dick, Sébastien Plissard et al.

III-V nanowires and nanowire optoelectronic devices

Yunyan Zhang, Jiang Wu, Martin Aagesen et al.

Direct nucleation, morphology and compositional tuning of InAs1xSbx nanowires on InAs (111) B substrates

Luna Namazi, Sepideh Gorji Ghalamestani, Sebastian Lehmann et al.

Nucleation and growth mechanism of self-catalyzed InAs nanowires on silicon

U P Gomes, D Ercolani, V Zannier et al. 


\title{
Tuning growth direction of catalyst-free InAs(Sb) nanowires with indium droplets
}

\author{
Heidi Potts, Nicholas P Morgan, Gözde Tütüncüoglu, Martin Friedl and \\ Anna Fontcuberta i Morral $^{1}$
}

Laboratoire des Matériaux Semiconducteurs, École Polytechnique Fédérale de Lausanne, 1015 Lausanne, Switzerland

E-mail: anna.fontcuberta-morral@epfl.ch

Received 2 October 2016, revised 16 November 2016

Accepted for publication 29 November 2016

Published 23 December 2016

\begin{abstract}
The need for indium droplets to initiate self-catalyzed growth of InAs nanowires has been highly debated in the last few years. Here, we report on the use of indium droplets to tune the growth direction of self-catalyzed InAs nanowires. The indium droplets are formed in situ on $\operatorname{InAs}(\mathrm{Sb})$ stems. Their position is modified to promote growth in the $\langle 11-2\rangle$ or equivalent directions. We also show that indium droplets can be used for the fabrication of InSb insertions in InAsSb nanowires. Our results demonstrate that indium droplets can initiate growth of InAs nanostructures as well as provide added flexibility to nanowire growth, enabling the formation of kinks and heterostructures, and offer a new approach in the growth of defect-free crystals.
\end{abstract}

S Online supplementary data available from stacks.iop.org/NANO/28/054001/mmedia

Keywords: nanowire, InAs, catalyst-free growth, molecular beam epitaxy, growth direction

(Some figures may appear in colour only in the online journal)

\section{Introduction}

InAs and InSb nanowires offer an interesting platform for fundamental studies and electronic applications [1-8]. For high performance devices it is important to achieve high material purity, a defect-free crystal structure [9], and flexibility in terms of dimensions, morphology and growth direction $[10,11]$. High purity nanowires can be obtained by molecular beam epitaxy (MBE) without a foreign catalyst, thereby avoiding the risk of impurity incorporation related to the catalyst particle $[12,13]$. However, a challenge remains concerning the presence of stacking defects in self-catalyzed InAs nanowires [14, 15]. One pathway towards defect-free crystals is the growth of ternary nanowires, as recently demonstrated by the growth of $\mathrm{InAs}_{1-x} \mathrm{Sb}_{x}$ nanowires $[16,17]$. Another approach to obtain defect-free nanowires would include a change of polarity [18] or growth direction [19], since the typical stacking defects observed in III-V nanowires are related to the formation of $\{111\}$ B planes [20]. In the case of InP nanowires, different crystalline growth

1 Author to whom any correspondence should be addressed. directions have been achieved by engineering the gold catalyst [21-25], or by spontaneous kinking in the case of selfcatalyzed nanowires [26]. The resulting wires in $\langle 100\rangle$ directions are defect-free [23]. Other directions, such as $\langle 112\rangle$ exhibit twin planes non-perpendicular to the nanowire axis $[20,27]$. The change in growth direction is not only relevant to suppress stacking defects, but also facilitates the integration with CMOS platforms where (100) wafers are typically used. Furthermore, in situ engineering of the droplet has opened many more perspectives, such as the growth of novel nanostructures, including nano-sheets [28-31] and nanocrosses [10, 32]. Branched nanostructures have received considerable attention as building blocks for braiding of Majorana Fermions [33]. In the case of self-catalyzed nanostructures droplet engineering is more difficult, and to the best of our knowledge, only one group has reported an in situ change of growth direction. In that work, branched GaAs nanowires were obtained by the accumulation of gallium on an InAs quantum dot on the nanowire side facet [34]. For InAs nanowires changing the growth direction by droplet engineering is particularly challenging, since there is still a debate as to whether InAs nanowires grow with or without an 
indium droplet [14, 15, 35]. Catalyst-free growth of InAs nanowires along the $\langle 100\rangle$ direction can be forced by using $\mathrm{SiO}_{2}$ nanotube templates, although this strategy does not prevent the formation of stacking defects [36]. As far as we know, template and catalyst-free InAs nanowires have so far been limited to the $\langle 111\rangle \mathrm{B}$ growth direction, and no in situ change of growth direction has been demonstrated yet.

\section{Results and Discussion}

In this work, we demonstrate that indium droplets can initiate and modify the growth direction of self-catalyzed InAs nanowires, even though in the typical growth regime indium droplets might not be present. Indium droplets are obtained either by annealing nanowires in vacuum or by direct deposition on the nanowires. We investigate the behavior of both InAs and InAsSb nanowires, and demonstrate that the indium droplet size and position can be tuned by changing the annealing time. The indium droplets can then be used as seeds to continue nanowire growth, thereby enabling the growth of L-shaped InAs nanostructures ('nano hockey sticks') as well as axial changes in composition. The L-shaped structures are particularly interesting because they give access to self-catalyzed InAs nanostructures grown in $\langle 11-2\rangle$ directions which adopt the crystal structure of the $\langle-1-1-1\rangle$ oriented host nanowire. In the case of pure InAs nanowires as a starting point, the resulting branches show stacking defects parallel to the growth direction. In the case of InAsSb nanowire host structures, branches with a very low defect density can be obtained.

We start by explaining the formation of indium droplets upon annealing of InAs nanowires. InAs nanowires are grown on GaAs(111)B substrates covered with $4.5 \mathrm{~nm}$ of HSQ oxide using a DCA P600 MBE system (more information about the nanowire growth can be found in [16]). After nanowire growth, all sources (In, As) are closed and the sample is kept at the growth temperature $\left(530^{\circ} \mathrm{C}\right)$ for a varying amount of time. We observe that arsenic evaporates at a higher rate compared to indium, as expected from the difference in vapor pressures. Since $\{111$ \} B facets are thermally less stable than $\{110\}$ facets, the evaporation occurs predominantly at the top facet of the nanowire, leading to the formation of an indium droplet on top of the wire. Representative scanning electron micrographs of the nanowires directly after growth and after being annealed for 5, 10 and $15 \mathrm{~min}$ are shown in figure 1. The indium droplets grow in size when increasing the annealing time, and slide down onto the side facets of the nanowire (figures 1(b), (c)) once they exceed a critical size. No significant change in diameter of the wires is observed as a function of the annealing time. This confirms that evaporation occurs mostly from the top facet and is negligible on the side facets, as opposed to what is observed in GaAs [37]. The droplets are located on the corner between two $\{1-10\}$ facets and stay close to the tip of the nanowire as shown in the $3 \mathrm{D}$ schematic in figure $1(\mathrm{~d})$. The pinning near the nanowire tip would be in agreement with the results on gold-catalyzed InSb nanosails [28], where it has been reported that a defectfree crystal structure allows for the droplet to slide down,
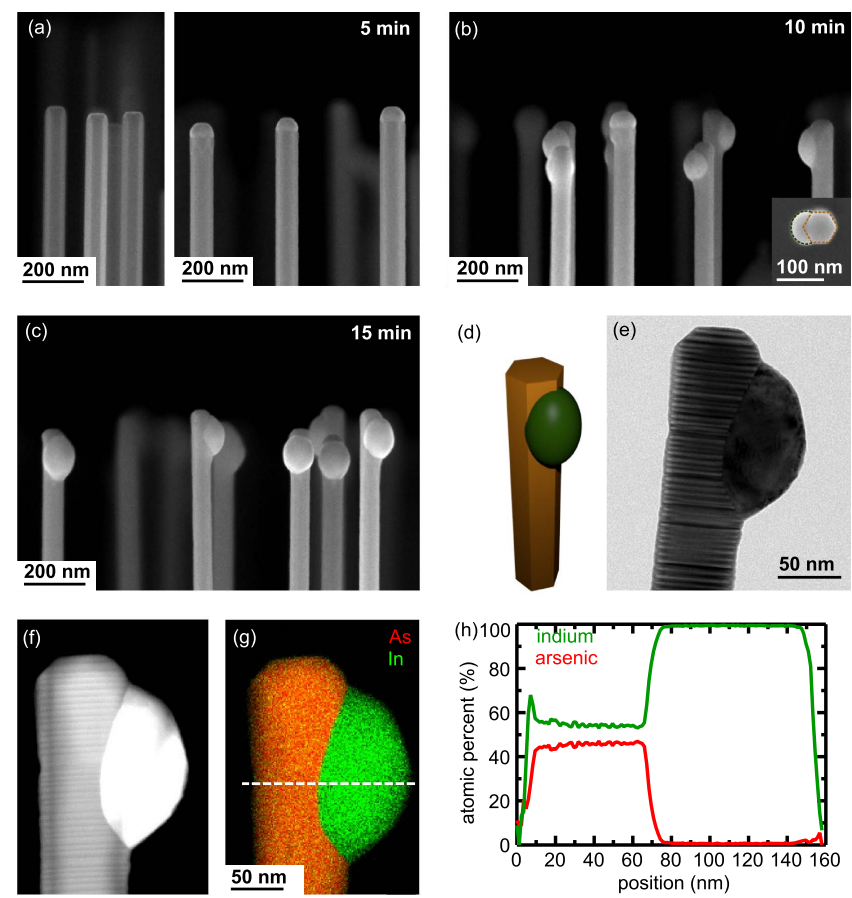

Figure 1. Droplet formation by annealing of InAs nanowires. (a) Cross sectional SEM micrographs of a standard InAs nanowire sample and after annealing for $5 \mathrm{~min}$ (b), (c) Nanowire samples which have been annealed for $10 \mathrm{~min}$, and $15 \mathrm{~min}$ respectively. Droplets are formed on the top facet of the nanowires and then slide down onto two of the $\{1-10\}$ facets when the droplets increase in size. Insert in (b) shows a top view SEM of a nanowire and droplet. (d) Schematic representation of the droplet position. (e) TEM micrograph of the tip of a nanowire with a droplet. (f), (g) STEMHAADF image and EDX map of the same nanowire. (h) Elemental composition along the line indicated in $(\mathrm{g})$, showing that the droplet is almost pure indium.

while stacking defects cause pinning of the droplet. In our case the host InAs nanowire shows a high density of stacking defects, as shown in the high resolution transmission electron micrograph (TEM) in figure 1(e), therefore pinning the droplet near the nanowire tip. The indium droplet is found to be amorphous after cooldown. We further performed energy dispersive $\mathrm{X}$-ray spectroscopy (EDX) in a scanning TEM to study the elemental composition of the droplet. Figures 1(f), (g) shows a high angular annular dark field image of a nanowire tip with a droplet, and an elemental map showing arsenic in red and indium in green. A linescan of the composition along the white dashed line is presented in figure $1(\mathrm{~h})$. The indium content is slightly above $50 \%$ on the nanowire. This suggests that a small layer of indium may accumulate at the nanowire surface. The formation of an indium droplet upon annealing of the nanowires in vacuum is also a critical point when discussing the exact growth mechanism (vapor-liquid-solid or vapor-solid) of InAs nanowires: the presence of an indium droplet is not necessarily related to vapor-liquid-solid since it can also be formed if the arsenic cell is closed before sufficient cooling of the nanowires.

Since the formation of a droplet depends on the preferred evaporation of arsenic at the nanowire tip, the question arises, 


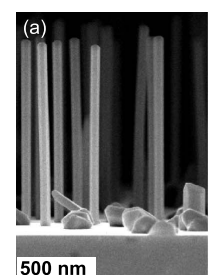

$00 \mathrm{~nm}$
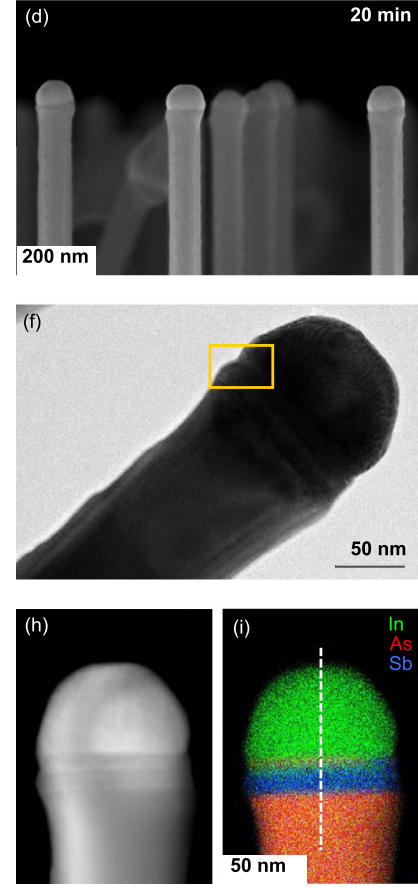
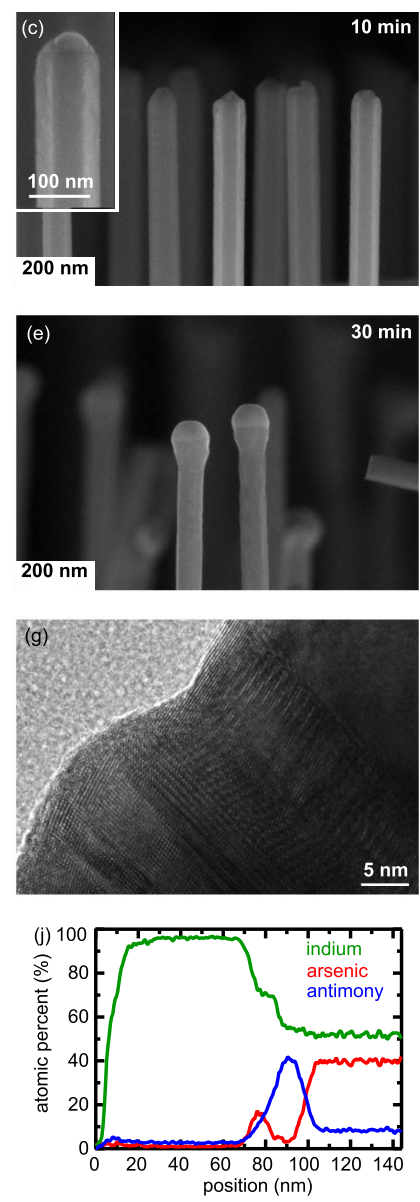

Figure 2. Droplet formation by annealing of InAsSb nanowires. (a) Standard InAsSb sample without annealing. (b) Nanowire sample which has been annealed for $10 \mathrm{~min}$ at $530^{\circ} \mathrm{C}$. (c)-(e) Nanowire samples which have been annealed at $500{ }^{\circ} \mathrm{C}$ for $10 \mathrm{~min}, 20 \mathrm{~min}$, and 30 min respectively. (f), (g) TEM micrographs of a nanowire with a droplet, showing an interference pattern due to crystals with a different lattice constant. (h), (i) STEM-HAADF image and EDX map of the same nanowire. (j) Elemental composition along the line indicated in (i), showing an InSb section between the InAsSb wire and the indium droplet.

how does this effect change in ternary materials (with two group $\mathrm{V}$ elements)? We chose to study InAsSb nanowires with an antimony content of approximately $20 \%$. The growth conditions and crystal structure analysis of InAsSb nanowires can be found in [16]. Figure 2(a) shows an SEM micrograph of an InAsSb nanowire sample before annealing. Similar to pure InAs nanowires, no droplet can be observed on the tip of standard InAsSb nanowires. Figure 2(b) shows an InAsSb sample which was annealed at $530{ }^{\circ} \mathrm{C}$ for $10 \mathrm{~min}$, in conditions similar to those that were found to lead to droplet formation for pure InAs nanowires. We observe that InAsSb is more sensitive to temperature; the nanowires evaporate at a much higher rate and the nanowire forest almost disappears, leaving indium droplets and crystallites on the substrate. Lowering the evaporation temperature by $30^{\circ} \mathrm{C}$ allows to form droplets on InAsSb nanowires. Figures 2(c)(e) corresponds to SEM micrographs of samples annealed for 10,20 and $30 \mathrm{~min}$ at $500{ }^{\circ} \mathrm{C}$. The droplet size increases at the beginning, but then stays constant after a certain time. The droplets do not slide down and instead the evaporation takes place mostly from the side facets, resulting in thinner nanowires with tapering towards the droplet. It is as if the droplet formed at the tip protects the top facet from evaporating further. Figures 2(f), (g) shows high resolution TEM investigations after cooldown. A Moiré pattern between the nanowire and the droplet is observed, suggesting an overlap of materials with a different lattice constant. STEM and EDX analysis of the nanowire tip and droplet are illustrated in figures 2(h)-(j). This analysis reveals the formation of a thin section of InSb just below a predominantly indium droplet. An InSb section is present in all annealed InAsSb samples (more details in supporting information). Since an InSb section is not observed in any as-grown nanowire sample, we assume that it is formed during cooldown; at the annealing temperature, the droplet consists of indium and antimony. During cooldown, the solubility of antimony in the indium droplet decreases, leading to a crystallization of InSb. We note that our results are qualitatively similar to the case of self-catalyzed GaAsSb nanowires, where a thin GaSb section has been reported [38]. A back-of-the-envelope calculation was done to estimate the amount of antimony dissolved in the indium droplet before cooldown. Assuming that the number of antimony atoms corresponds to the InSb section of $19 \pm 5 \mathrm{~nm}$ in height, we get an antimony concentration of $15 \pm 4 \%$ in the indium droplet before cooldown. This value is comparable with self-catalyzed InSb nanowires grown by MOVPE, where an antimony concentration of up to $20 \%$ was measured in the droplet [39].

We turn now to the use of indium droplets as seed particles to drive InAs nanowire growth in new growth directions. After growing the InAs nanowires, we anneal them to form the indium droplets at the top or side facets of the nanowires, and then continue growth. For the second part of the growth we use typical InAs nanowire growth conditions in terms of substrate temperature and indium flux $\left(530{ }^{\circ} \mathrm{C}\right.$ and $1.4 \times 10^{-7}$ Torr indium beam equivalent pressure (BEP)), while the arsenic BEP is lower and was varied between 2.5 and $7 \times 10^{-7}$ Torr. Figures $3(\mathrm{a})-(\mathrm{c})$ show representative SEM micrographs of InAs samples annealed for $5 \mathrm{~min}$, $10 \mathrm{~min}$ and $15 \mathrm{~min}$ which were then subjected to further InAs growth $(1 \mathrm{~h})$ at an arsenic BEP of $5 \times 10^{-7}$ Torr. Figure 3(a) shows that after only $5 \mathrm{~min}$ annealing, growth mainly continues in the original $\langle-1-1-1\rangle$ nanowire growth direction (the expected droplet position is marked with a dashed line). After 10 min annealing we observe the growth of L-shaped structures as shown in figure 3(b). Those structures are always perpendicular to the host nanowire, similar to the results by Suyatin et al, where kinked InAs nanowires were grown by a second gold deposition step and chemical beam epitaxy [40]. In figure 3(b) we also note that not all the wires form branches, as some wires also continue growing in the axial direction. We attribute this variation of shapes to the presence of indium droplets both at the nanowire tip and side facets after $10 \mathrm{~min}$ annealing. Growth after $15 \mathrm{~min}$ annealing results in $100 \% \mathrm{~L}$-shaped nanostructures. This is in agreement with the $100 \%$ yield of droplet formation at the intersection of two nanowire side facets. It can therefore be concluded that the 

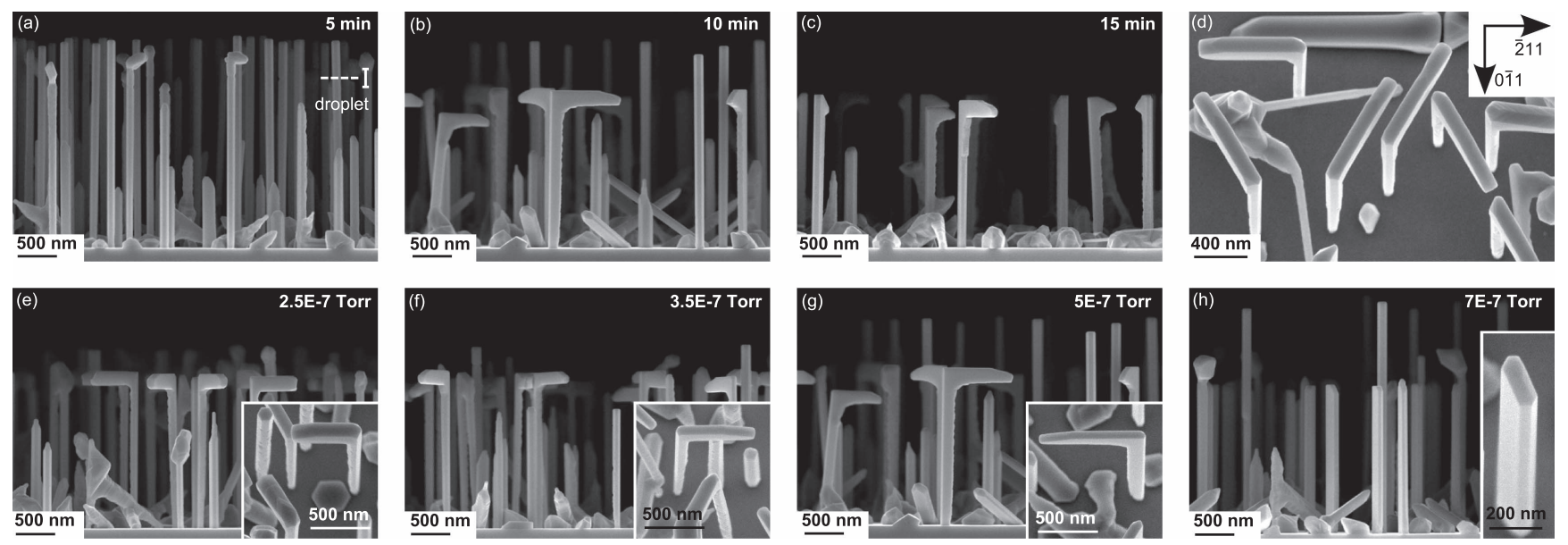

Figure 3. Growth of L-shaped InAs nanostructures. (a)-(c) Cross sectional SEM micrographs of InAs nanowire samples that have been annealed for $5 \mathrm{~min}, 10 \mathrm{~min}$ and $15 \mathrm{~min}$ respectively. The growth of the branch was performed under $5 \times 10^{-7}$ Torr arsenic flux for $1 \mathrm{~h}$. We observe that the growth direction after annealing is determined by the droplet position. (d) Top view image ( $15^{\circ}$ tilted) of the sample which was annealed for $10 \mathrm{~min}$, showing the crystalline direction of the branches. (e)-(h) Cross sectional SEM micrographs and top view images $\left(15^{\circ}\right.$ tilted $)$ of nanowire samples that have been annealed for 10 min and growth was re-initiated under an arsenic BEP of $2.5 \times 10^{-7}$ Torr, $3.5 \times 10^{-7}$ Torr, $5 \times 10^{-7}$ Torr, and $7 \times 10^{-7}$ Torr, respectively. We observe that growth in $\langle 11-2\rangle$ direction is favored at low arsenic pressure, while flat nanostructures are observed at high arsenic pressure.

droplets act as seed particles to re-initiate growth following the vapor-liquid-solid (VLS) mechanism. At this point it is still unclear how long the droplet survives and whether or not the mechanism changes to vapor-solid (VS). Figure 3(d) shows a top view image $\left(15^{\circ}\right.$ tilted $)$ of the sample presented in (b). It can be observed that the growth direction of the L-shaped structures corresponds to one of the $\langle 11-2\rangle$ directions, being in agreement with the positioning of the indium droplets at the corners between two $\{110\}$ facets. Interestingly the $\langle 11-2\rangle$ direction is also the growth direction for which the growth of membranes and nanowires with elongated cross sections is possible in the case of GaAs in patterned silicon dioxide on GaAs(111)B [41, 42].

We will now discuss the role of the arsenic flux in the growth of $\langle 11-2\rangle$ oriented nanostructures. In figures 3(e)-(h) we provide SEM micrographs of samples grown with an increasing value of arsenic BEP, after annealing the InAs stems for a duration of $10 \mathrm{~min}$. The exact values of arsenic BEP are $2.5 \times 10^{-7}$ Torr, $3.5 \times 10^{-7}$ Torr, $5 \times 10^{-7}$ Torr, and $7 \times 10^{-7}$ Torr for samples (e)-(h), respectively (note that (b) and (g) are the same sample). We observe that increasing the arsenic flux significantly increases the growth both in the $\langle-1-1-1\rangle$ direction for the linear nanowires and in the $\langle 11-2\rangle$ direction for the L-shaped nanostructures (figures 3(e)-(g)). For very high arsenic flux growth in the $\langle 11-2\rangle$ direction stops to be favorable, and nanowires with elongated cross sections are observed as shown in figure 3(h). Note that the threshold is lower than the arsenic BEP which is used for the growth of typical InAs nanowires $\left(1.9 \times 10^{-6}\right.$ Torr $)$. Time series reveal that the flat membranes form gradually; it is as if the droplets favor growth on the side facet they were initially sitting on (more information available in supporting information). The observed mechanism stands in contrast to gold catalyzed nanowires, for which flat nanowires were reported due to a downward movement of the catalyst particle [43].
In figure 4 we show how re-initiating growth with an indium droplet affects the crystal structure. Previously it has been suggested that the presence of liquid indium could favor the growth of defect-free Wurtzite [44], and it has been demonstrated that controlling the droplet is crucial for the growth of defect-free GaAs nanowires [45, 46]. We start by analyzing the crystal structure of the L-shaped nanostructures. An SEM micrograph of the sample is shown in figure 4(a). Figures 4(b), (c) show a low resolution TEM micrograph and the corresponding diffraction pattern of an InAs branch. High resolution TEM micrographs of different parts of the sample are shown in figures 4(d), (e). We observe that the crystal structure is polytypic, as is commonly observed for self-catalyzed InAs nanowires. Twins form perpendicular to the nanowire axis, as is also commonly observed for nanowires grown in the $\langle 111\rangle$ direction [20]. Comparing a high-resolution TEM micrograph of the corner (figure 4(d)) with the end of the branch (figure 4(e)) we observe that the defects of the host nanowire propagate all along the horizontal part, being parallel to the growth direction. The $\langle 11-2\rangle$ oriented part of the L-shaped structure therefore adopts the polytypic crystal structure from the host InAs nanowire. This observation stands in contrast to the work by Plissard et al [10], where the horizontal InSb nanowire has no epitaxial relation with the InP nanowire stem. We also study the crystal structure of the sample shown in figure 4(f), where the growth continues in axial direction. Figures $4(\mathrm{~g})$, (h) show a low resolution TEM micrograph and the corresponding diffraction pattern. We observe that the general crystal structure is polytypic. However, in many wires we observe a thick pure zinc blende (ZB) section $800 \mathrm{~nm}$ below the tip of the nanowire, as shown in figures 4(i), (j). The location of the pure ZB region corresponds approximately to the expected position where the growth was re-initiated after the droplet formation. Since the existence of a pure $\mathrm{ZB}$ region in the middle of the 

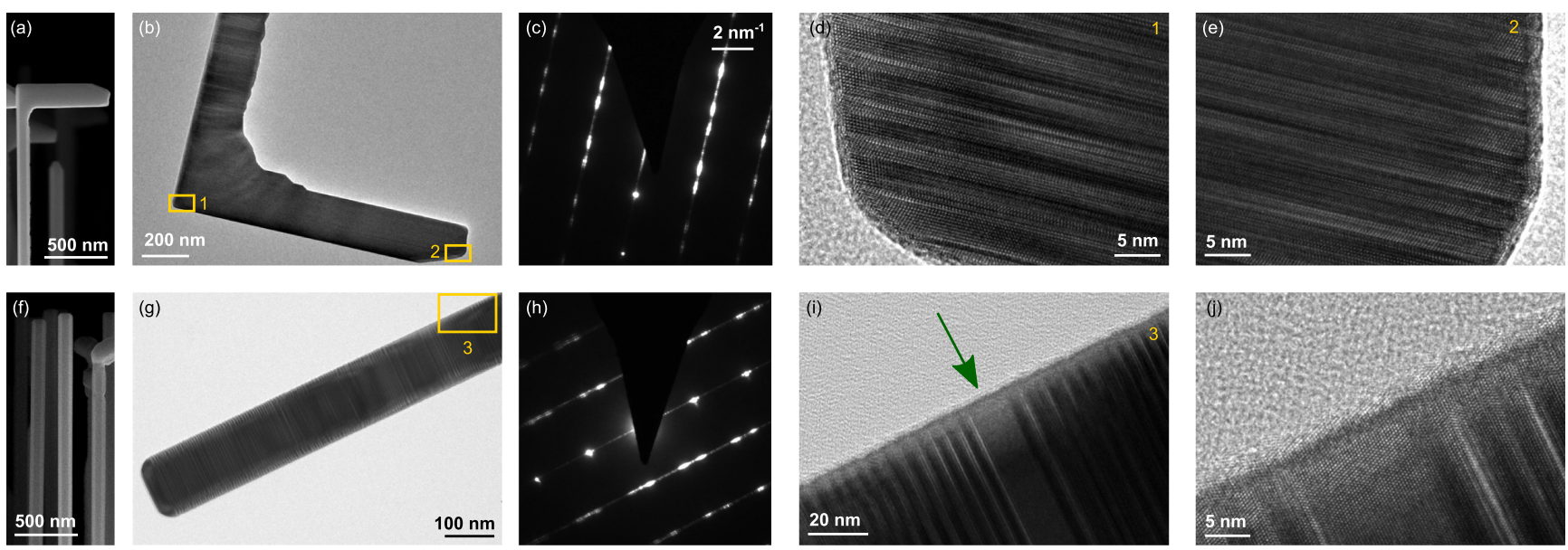

Figure 4. Crystal structure of InAs nanostructures grown with a droplet. (a) SEM micrograph of an L-shaped nanostructure. (b)-(e) TEM micrographs showing that the branch adopts the polytypic crystal structure of the InAs host nanowire. (f) SEM micrograph of an InAs nanowire sample where the upper part of the nanowire was grown after droplet formation (g)-(j) TEM micrographs showing the polytypic crystal structure with a pure ZB section of $10 \mathrm{~nm}$.

polytypic InAs crystal structure is very unusual, we attribute it to the effect of the indium droplet and the VLS growth mechanism. After the pure ZB section the nanowire continues with the standard polytypic crystal structure, suggesting that the droplet is readily consumed and the growth proceeds droplet-free. Comparing our results to the findings of Biermanns et al [44], we agree that the existence of an indium droplet supports the suppression of twinning defects. One should note, however, that in our case the resulting crystal structure is $\mathrm{ZB}$, while the original paper reported on wurtzite (WZ) stacking.

We go now a step further in the fabrication of hierarchical structures, and investigate the formation of droplets when annealing L-shaped nanostructures. Figure 5(a) shows a sample which has been annealed for $10 \mathrm{~min}$ at $530{ }^{\circ} \mathrm{C}$. Different positions of the indium droplets can be observed. Figures 5(b)-(e) shows a sample where growth was re-initiated after the second droplet formation step. Different nanostructure shapes can be observed, including thicker L-shaped structures, longer branches, and nano-bridges. The nano-bridges are particularly interesting since the second 'leg', which is formed after the second annealing step, grows in $\langle 111\rangle$ A direction. A-polar growth is very untypical for selfcatalyzed arsenide nanowires, and has only recently been observed in the case of InAs growing around $\mathrm{Si} / \mathrm{SiO}_{2}$ nanotubes [47]. Our results confirm that it is possible under certain conditions. A low resolution TEM micrograph of a nanobridge is shown in figure 5(f). A high resolution TEM micrograph of the host nanowire is presented in figure $5(\mathrm{~g})$, showing the standard polytypic crystal structure. In the high resolution TEM micrograph of the second 'leg' (figure 5(f)) we can observe a $20 \mathrm{~nm}$ thick pure $\mathrm{ZB}$ region. We attribute this pure phase section to the presence of a droplet when reinitiating growth. The rest of the second leg shows polytypism, similar to standard InAs nanowires. Please note that nano-bridges can also be formed when two L-shaped nanostructures merge during growth. However, in such a case the crystal structure of the horizontal part is not continuous (more
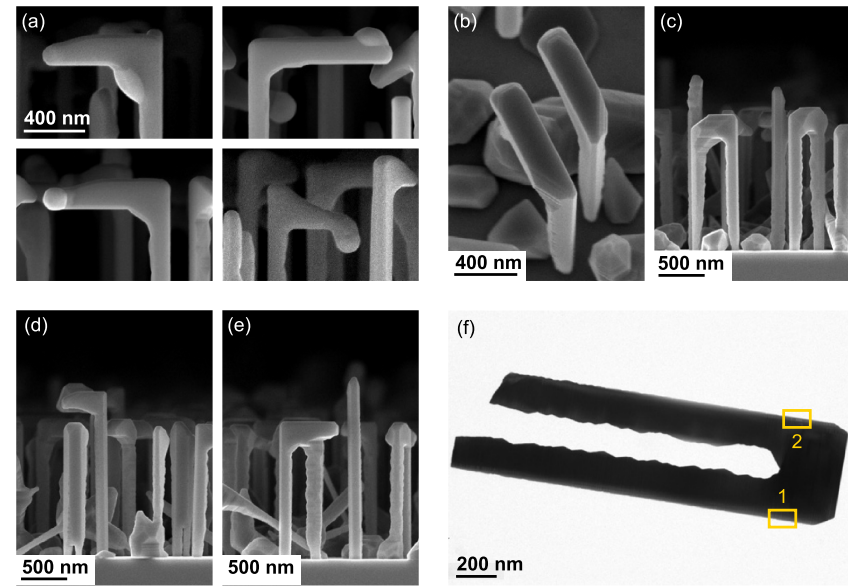

(f)
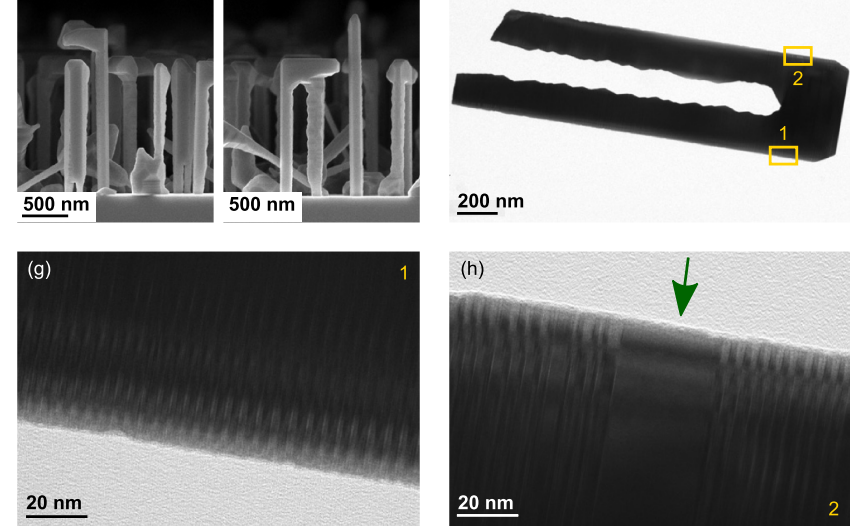

$200 \mathrm{~nm}$

Figure 5. Droplet formation and growth re-initiation on L-shaped nanostructures. (a) Cross sectional SEM micrographs of a sample after annealing, showing different droplet positions on the L-shaped nanostructures. (b)-(e) Top view (15 $5^{\circ}$ tilt) and cross sectional SEM micrographs of a sample which was first annealed and then had growth continue to form a variety of different nanostructure shapes. (f)-(h) TEM micrographs of a nano-bridge. Both legs have a similar polytypic crystal structure. The upper leg, which was grown after the second droplet formation, shows a $20 \mathrm{~nm}$ pure ZB insertion.

details in supporting information). We conclude that the annealing and re-initiation process can be repeated multiple times in order to grow more sophisticated hierarchical structures. Further research is necessary to achieve control over the droplet position during consecutive annealing steps.

Next we present re-initiation of InAs growth in the case of InAsSb nanowires after droplet formation. We have seen 


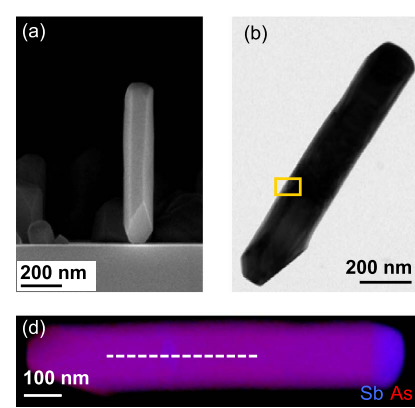

(f)
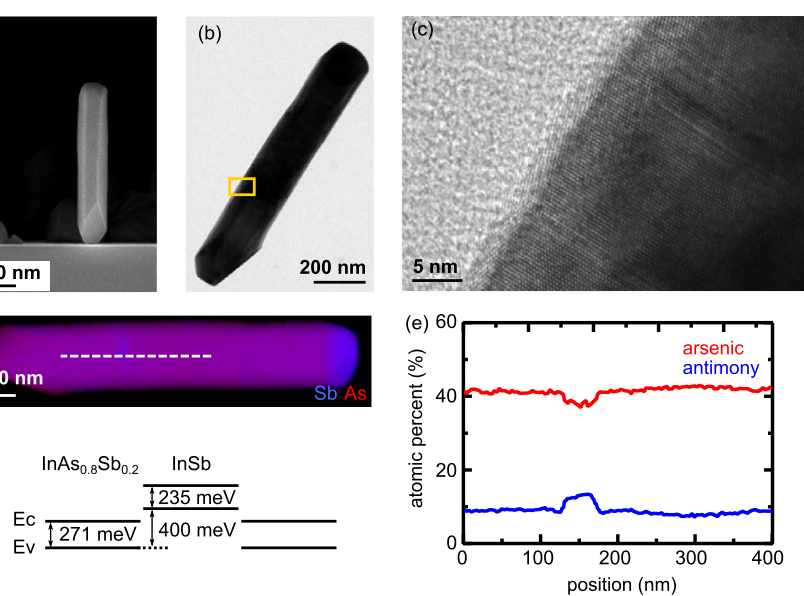

Figure 6. Growth of an InSb insertion in an InAsSb nanowire. (a) SEM micrograph of a nanowire sample which has an embedded InSb region. (b), (c) TEM micrographs showing the change in crystal structure due to the InSb section. (d), (e) EDX map and linescan confirmation of the existence of an InSb region. (f) Band alignment of the $\mathrm{InSb}$ section embedded in InAsSb at $4 \mathrm{~K}$.

that in this case the droplet forms at the nanowire tip. When re-initiating growth after droplet formation all nanowires continue to grow vertically. Interestingly, no InSb section can be observed at the interface between InAsSb and InAs, suggesting that the InSb is formed during cooldown (more information is available in the supporting information). In order to grow InSb sections embedded in InAsSb samples we therefore investigate nanowire growth with a cooldown step after droplet formation. Figure 6(a) shows an SEM micrograph of a nanowire sample which was first annealed in order to form a droplet, then cooled down to form an InSb section and then overgrown with InAsSb. TEM micrographs of the sample are shown in figures 6(b), (c). We can observe a change in crystal structure within the otherwise defect-free InAsSb nanowire. The EDX map and linescan shown in figures 6(d), (e) confirm the existence of an InSb section of approximately $20 \mathrm{~nm}$ in length and $70 \mathrm{~nm}$ in diameter. The band alignment of an InSb section within $\mathrm{InAs}_{0.8} \mathrm{Sb}_{0.2}$ is shown in figure 6(f). The bandgap energy and band offset was calculated using the bowing parameters of [48]. We suggest that the InSb insertion can be used as a quantum dot memory for holes, or as an electron transport barrier after etching away the InAsSb radial overgrowth.

To add more flexibility to the choice of host structure, we also investigate the possibility to deposit indium on nanowires in situ after growth. This is achieved by opening the indium flux after growth while keeping arsenic closed. In order to avoid evaporation of the nanowires, the sample temperature is lowered to $480{ }^{\circ} \mathrm{C}\left(430^{\circ} \mathrm{C}\right)$ for $\mathrm{InAs}$ (InAsSb) nanowires. Figure 7(a) shows an SEM micrograph of an InAs nanowire sample where indium has been deposited for $3 \mathrm{~min}$. We observe the formation of indium droplets on the $\{110\}$ facets of the nanowires. In this case the droplets are formed at the base of the nanowires, without wetting the HSQ oxide. Figures 7(b), (c) show SEM micrographs of an InAs sample where indium droplets were deposited and then growth was continued with the standard parameters for the growth of branches $\left(3.5 \times 10^{-7}\right.$ Torr arsenic BEP). We will refer to this sample as InAs-In-InAs, according to the different steps during growth. We observe that the deposited indium acts as a seed particle to continue growth in $\langle 11-2\rangle$ direction, similar to the samples presented above. Here, the horizontal part of the L-shaped structure is at the base of the nanowires, due to the location of the indium droplets. Figures 7(d)-(f) show SEM micrographs of the corresponding experiments on InAsSb nanowires as host structures. We observe that droplets also form at the base of the nanowires, resulting in L-shaped structures where the horizontal part is close to the substrate. Note that under the conditions presented here, not all nanowires have an indium droplet after indium deposition. Therefore there are also nanowires without the horizontal part after re-initiating growth. Figures $7(\mathrm{~g})-(\mathrm{k})$ shows a crystal structure and composition analysis of the InAs-In-InAs sample. We find that the sample horizontal part of the nanostructure inherits the polytypic crystal structure of the host nanowire, similar to the results presented in figure 4 . The STEM-EDX map shown in figure 7(i) confirms that the nanostructure is pure InAs. Figures 7(1)-(p) shows a crystal structure and composition analysis of the InAsSb-In-InAs sample. The diffraction pattern (figure $7(\mathrm{~m})$ ), which was acquired on the horizontal part, shows that the structure is pure ZB. The STEM-EDX analysis in figure 7(n) confirms that the host nanostructure is InAsSb, while the horizontal part is InAs. At the tip of the horizontal InAs part we note a few rotational twins, as shown in the high resolution TEM micrographs in figures $7(\mathrm{o})$, (p). The samples presented here show that depositing indium on a host structure allows to form heterostructures, where the horizontal part adopts the crystal structure of the host nanowire all along the branch. However, we need to note that the horizontal part of the structure can in some cases also exhibit defects which do not propagate from the host nanowire and suggest a change of the growth front (more information available in supporting information). We attribute this finding to the close proximity of the substrate.

Finally, we also study the electrical properties of the L-shaped nanostructures grown by the annealing method. Electrical contacts to the nanostructure were defined by e-beam lithography, followed by argon milling to remove the native oxide, and then sputtering of chromium/gold. A detailed description of the process can be found in [16]. Figure 8(a) shows an SEM micrograph of a device allowing 2-point electrical measurements of the 'main', 'branch' and 'corner' sections of the L-shaped structure. Figure 8(b) shows an AFM image of another such device with a linescan across the branch section of the device, showing it to have an approximately rectangular cross section. Figure 8(c) shows representative $I-V$ curve behavior of such devices. The linearity indicates ohmic contact behavior. For the devices studied, the $I-V$ characteristics were used to estimate the conductivity of the different parts of the nanostructure based on AFM height and SEM length/width measurements. The average conductivity of the branches was found to be $81 \pm 23 \mathrm{~S} \mathrm{~cm}^{-1}$ (for four devices), which is comparable to standard $\langle 111\rangle \mathrm{B}$ self-catalyzed InAs nanowires [16]. 

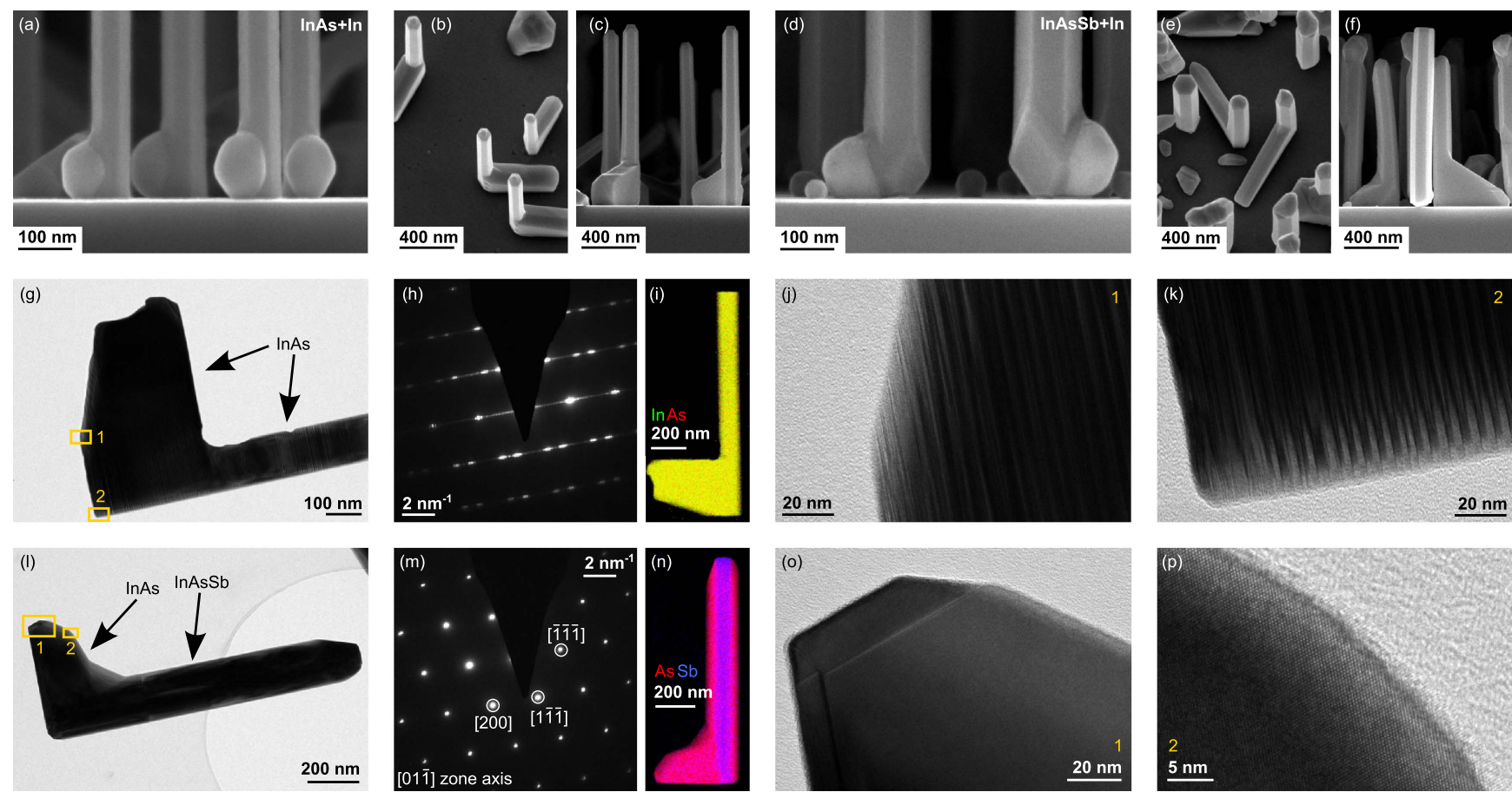

$20 \mathrm{~nm}$

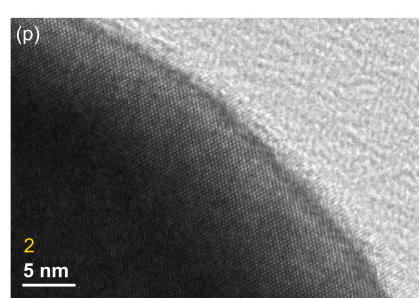

Figure 7. Deposition of indium droplets on $\operatorname{InAs}(\mathrm{Sb})$ nanowires to re-initiate growth. (a) InAs nanowires with indium deposition for 3 min (b), (c) Top view (15 tilt) and cross sectional SEM micrograph of an InAs-In-InAs sample. (d) InAsSb nanowires with indium deposition for 10 min. (e), (f) Top view (15 tilt) and cross sectional SEM micrograph of an InAsSb-In-InAs sample. (g) Low resolution TEM micrograph of an InAs-In-InAs nanostructure. (h) Diffraction pattern acquired on the horizontal part. (i) STEM-EDX map of the InAs-InInAs nanostructure. (j), (k) High resolution TEM micrographs of the regions indicated by the yellow rectangles. (l)-(p) Similar analysis on an InAsSb-In-InAs nanostructure.
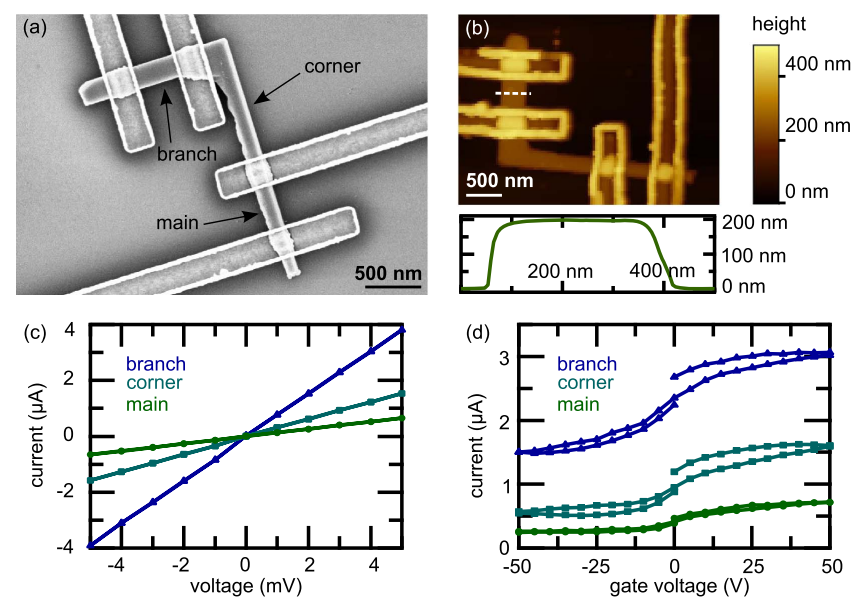

Figure 8. Electrical properties of L-shaped InAs nanostructures. (a) SEM micrograph of an electrical device. (b) AFM image and linescan of another electrical device, showing vertical profile of the branch section. (c) Representative $I-V$ curve behavior of all three sections of a device as shown above. (d) Gate response of a typical device for a fixed source-drain voltage of $3 \mathrm{mV}$.

Figure 8(d) shows representative behavior of these devices when sweeping back-gate voltage for a fixed source-drain voltage of $3 \mathrm{mV}$. It was found that, in general, all parts of the device show n-type gate response, though the intensity of the response varied significantly. None of the devices could be turned off completely, probably due to charge screening of the gate by the relatively thick devices. Upon inspection of the devices after electrical testing, some of the devices appeared melted and amorphous. The results of the melted devices were not included in the conductivity calculation. At this point it is still unclear at which point during the measurements the melting occurred. More information on this phenomenon is given in the supporting information section.

\section{Conclusions}

In conclusion, we have demonstrated how to change the growth direction of self-catalyzed InAs nanostructures by in situ formation and manipulation of indium droplets. We have presented a detailed study on how to obtain indium droplets by annealing both for InAs and InAsSb nanowires. The indium droplets can then be used to initiate growth in different crystalline directions, resulting in linear or L-shaped nanostructures, depending on the position of the droplet. The conductivity of the branches was found to be roughly comparable to standard InAs nanowires, and all parts of the nanostructure show n-type gate response. Showing that indium droplets can be formed upon annealing further offers a new perspective when discussing whether or not a droplet is present during the growth of self-catalyzed InAs nanowires, and how the presence of liquid indium affects the crystal structure. In the case of InAsSb nanowires, our approach can be used to fabricate InSb sections within InAsSb wires, which are expected to act as quantum dot memories for holes. Furthermore, we demonstrate the flexibility of this technique by deposition of indium on $\operatorname{InAs}(\mathrm{Sb})$ after growth. Applying 
the indium deposition to different host nanostructures will allow for the growth of heterostructures or defect-free branches. Our results contribute to the understanding of the role of indium droplets in self-catalyzed InAs nanowire growth and offer a whole spectrum of new opportunities for indium-based nanostructures, including branched nanostructures, and the possibility to control defect formation. Demonstrating a change of growth direction for catalyst-free InAs nanowires also paves the route towards $\langle 100\rangle$ oriented nanowires, which would facilitate the integration with CMOS technologies.

\section{Acknowledgments}

The authors acknowledge the SNF for financial support via the project 200021_169908 and through the NCCR QSIT. We would like to thank Jean-Baptiste Leran for help with MBE maintenance, Thomas La Grange, Duncan Alexander and Marco Cantoni from CIME for TEM support, and Philippe Caroff and Frank Glas for fruitful discussions.

\section{References}

[1] Mourik V, Zuo K, Frolov S M, Plissard S R, Bakkers E P A M and Kouwenhoven L P 2012 Signatures of majorana fermions in hybrid superconductor-semiconductor nanowire devices Science 251003

[2] Brongersma M L, Cui Y and Fan S 2014 Light management for photovoltaics using high-index nanostructures Nat. Mater. 13 451-60

[3] del Alamo J A 2011 Nanometre-scale electronics with III-V compound semiconductors Nature 479317

[4] Li Y, Qian F, Xiang J and Lieber C M 2006 Nanowire electronic and optoelectronic devices Mater. Today 918

[5] Takei K, Takahashi T, Ho J C, Ko H, Gillies A G, Leu P W, Fearing R S and Javey A 2010 Nanowire active-matrix circuitry for low-voltage macroscale artificial skin Nat. Mater. 9821

[6] Dayeh S H, Aplin D P R, Zhou X, Yu P K L, Yu E T and Wang D 2007 High electron mobility InAs nanowire fieldeffect transistors Small 3326

[7] Deng M T, Yu C L, Huang G Y, Larsson M, Caroff P and $\mathrm{Xu}$ H Q 2012 Anomalous zero-bias conductance peak in a $\mathrm{Nb}-\mathrm{InSb}$ nanowire-Nb hybrid device Nano Lett. 126414

[8] Kang S L N, Fan D X, Wang L B, Huang Y Q, Caroff P and $\mathrm{Xu}$ H Q 2016 Coherent charge transport in ballistic InSb nanowire Josephson junctions Sci. Rep. 624822

[9] Thelander C, Caroff P, Plissard S, Dey A W and Dick K A 2011 Effects of crystal phase mixing on the electrical properties of InAs nanowires Nano Lett. 112424

[10] Plissard S R et al 2013 Formation and electronic properties of InSb nanocrosses Nat. Nanotechnol. 8859

[11] Dick K A, Deppert K, Larsson M W, Martensson T, Seifert W, Wallenberg L R and Samuelson L 2004 Synthesis of branched 'nanotrees' by controlled seeding of multiple branching events Nat. Mater. 3380

[12] Bar-Sadan M, Barthel J, Shtrikman H and Houben L 2012 Direct imaging of single $\mathrm{Au}$ atoms within $\mathrm{GaAs}$ nanowires Nano Lett. 122352

[13] Koto M, Marshall A F, Goldthorpe I A and McIntyre P C 2010 Gold-catalyzed vapour-liquid-solid germanium-nanowire nucleation on porous silicon Small 61032
[14] Grap T, Rieger T, Blömers C, Schäpers T, Grützmacher D and Lepsa M I 2013 Self-catalyzed VLS grown InAs nanowires with twinning superlattices Nanotechnology 24335601

[15] Dimakis E, Lähnemann J, Jahn U, Breuer S, Hilse M, Geelhaar L and Riechert H 2011 Self-assisted nucleation and vapor-solid growth of InAs nanowires on bare $\mathrm{Si}(111)$ Cryst. Growth Des. 114001

[16] Potts H, Friedl M, Amaduzzi F, Tang K, Tütüncüoglu G, Matteini F, Alarcon Llado E, McIntyre P C and Fontcuberta i Morral A 2016 From twinning to pure zincblende catalyst-free $\mathrm{InAs}(\mathrm{Sb})$ nanowires Nano Lett. 16637

[17] Sourribes M J L, Isakov I, Panfilova M, Liu H and Warburton P A 2014 Mobility enhancement by Sb-mediated minimisation of stacking fault density in InAs nanowires grown on silicon Nano Lett. 141643

[18] Wacaser B A, Deppert K, Karlsson L S, Samuelson L and Seifert W 2006 Growth and characterization of defect free GaAs nanowires J. Cryst. Growth 287504

[19] Yuan X, Caroff P, Wong-Leung J, Fu L, Tan H H and Jagadish C 2015 Tunable polarity in a III-V nanowire by droplet wetting and surface energy engineering $A d v$. Mater. 276096

[20] Conesa-Boj S, Zardo I, Estrade S, Wei L, Alet P J, Roca I Cabarrocas P, Morante J R, Peir F, Fontcuberta i Morral A and Arbiol J 2010 Defect formation in Ga-catalyzed silicon nanowires Cryst. Growth Des. 101534

[21] Krishnamachari U, Borgstrom M, Ohlsson B J, Panev N, Samuelson L, Seifert W, Larsson M W and Wallenberg L R 2004 Defect-free InP nanowires grown in [001] direction on InP(001) Appl. Phys. Lett. 852077

[22] Fonseka H A, Caroff P, Wong-Leung J, Ameruddin A S, Tan H H and Jagadish C 2014 Nanowires grown on InP (100): growth directions, facets, crystal structures and relative yield control ACS Nano 86945

[23] Wan J, Plissard S, Hocevar M, Vu T T T, Zehender T, Immink G G W, Verheijen M A, Haverkort J and Bakkers E P A M 2012 Position-controlled [100] InP nanowire arrays Appl. Phys. Lett. 100053107

[24] Wan J, Plissard S R, Verheijen M A, Feiner L-F, Cavalli A and Bakkers E P A M 2013 Reversible switching of InP nanowire growth direction by catalyst engineering Nano Lett. 133802

[25] Mikkelsen A, Eriksson J, Lundgren E, Andersen J N, Weissenrieder J and Seifert W 2005 The influence of lysine on $\mathrm{InP}(001)$ surface ordering and nanowire growth Nanotechnology 162354

[26] Yan X, Zhang X, Li J, Wu Y and Ren X 2015 Self-catalyzed growth of pure zinc blende $\langle 110\rangle \mathrm{InP}$ nanowires Appl. Phys. Lett. 107023101

[27] Li Z-A et al 2011 Planar-defect characteristics and crosssections of $\langle 001\rangle,\langle 111\rangle$, and $\langle 112\rangle$ InAs nanowires $J$. Appl. Phys. 109114320

[28] Kelrich A, Sorias O, Calahorra Y, Kauffmann Y, Gladstone R, Cohen S, Orenstein M and Ritter D 2016 InP nanoflag growth from a nanowire template by in situ catalyst manipulation Nano Lett. 162837

[29] Pan D, Fan D X, Kang N, Zhi J H, Yu X Z, Xu H Q and Zhao J H 2016 Free-standing two-dimensional singlecrystalline InSb nanosheets Nano Lett. 16834

[30] De La Mata M, Leturcq R, Plissard S R, Rolland C, Magén C, Arbiol J and Caroff P 2016 Twin-induced InSb nanosails: a convenient high mobility quantum system Nano Lett. 16825

[31] Aagesen M, Johnson E, Sorensen C B, Mariager S O, Feidenhans'l R, Spiecker E, Nygard J and Lindelof P E 2007 Molecular beam apitaxy growth of free-standing planeparallel InAs nanoplates Nat. Nanotechnol. 2761 
[32] Car D, Wang J, Verheijen M A, Bakkers E P A M and Plissard S R 2014 Rationally designed single-crystalline nanowire networks Adv. Mater. 264875

[33] Alicea J, Oreg Y, Refael G, von Oppen F and Fisher M P A 2011 Non-Abelian statistics and topological quantum information processing in 1D wire networks Nat. Phys. 7412

[34] Yu Y et al 2013 Single InAs quantum dot grown at the junction of branched gold-free GaAs nanowire Nano Lett. 131399

[35] Gomes U P, Ercolani D, Zannier V, David J, Gemmi M, Beltram F and Sorba L 2016 Nucleation and growth mechanism of self-catalyzed InAs nanowires on silicon Nanotechnology 27255601

[36] Borg M, Schmid H, Moselund K E, Signorello G, Gignac L, Bruley J, Breslin C, Das Kanungo P, Werner P and Riel H 2014 Vertical III-V nanowire device integration on $\mathrm{Si}(100)$ Nano Lett. 141914

[37] Loitsch B et al 2015 Tunable quantum confinement in ultrathin, optically active semiconductor nanowires via reverse-reation growth Adv. Mater. 272195

[38] Conesa-Boj S, Kriegner D, Han X-L, Plissard S, Wallart X, Stangl J, Fontcuberta i Morral A and Caroff P 2014 Goldfree ternary III-V antimonide nanowire arrays on silicon: twin-free down to the first bilayer Nano Lett. 14326

[39] Mandl B, Dick K A, Kriegner D, Keplinger M, Bauer G, Stangl J and Deppert K 2011 Crystal structure control in Aufree self-seeded InSb wire growth Nanotechnology 22 145603

[40] Suyatin D B, Sun J, Fuhrer A, Wallin D, Fröberg L E, Karlsson L S, Maximov I, Wallenberg L R, Samuelson L and Xu H Q 2008 Electrical properties of self-assembled branched InAs nanowire junctions Nano Lett. 41100
[41] Tutuncuoglu G, de la Mata M, Deiana D, Potts H, Matteini F, Arbiol J and Fontcuberta i Morral A 2015 Towards defectfree $1 \mathrm{D} \mathrm{GaAs} / \mathrm{AlGaAs}$ heterostructures based on GaAs nanomembranes Nanoscale 719453

[42] Foster A P, Bradley J P, Gardner K, Krysa A B, Royall B, Skolnick S and Wilson L R 2015 Linearly polarized emission from an embedded quantum dot using nanowire morphology control Nano Lett. 151559

[43] Paladugu M, Zou J, Guo Y-N, Auchterlonie G J, Joyce H J, Gao Q, Tan H H, Jagadish C and Kim Y 2007 Novel growth phenomena observed in axial InAs/GaAs nanowire heterostructures Small 31873

[44] Biermanns A, Dimakis E, Davydok A, Sasaki T, Geelhaar L, Takahasi M and Pietsch U 2014 Role of liquid indium in the structural purity of wurtzite InAs nanowires that grow on Si(111) Nano Lett. 146878

[45] Jacobsson D, Panciera F, Tersoff J, Reuter M C, Lehmann S, Hofmann S, Dick K A and Ross F M 2016 Interface dynamics and crystal phase switching in GaAs nanowires Nature 531317

[46] Balaghi L, Tauchnitz T, Hübner R, Bischoff L, Schneider H, Helm M and Dimakis E 2016 Droplet-confined alternate pulsed epitaxy of GaAs nanowires on $\mathrm{Si}$ substrates down to CMOS-compatible temperatures Nano Lett. 164032

[47] Vukajlovic-Plestina J, Dubrovskii V, Tutuncuoglu G, Potts H, Ricca R, Meyer F, Matteini F, Leran J-B and Fontcuberta i Morral A 2016 Molecular beam epitaxy of InAs nanowires in $\mathrm{SiO}_{2}$ nanotube templates: challenges and prospects for integration of III-Vs on Si Nanotechnology 27 455601

[48] Wei S H and Zunger A 1995 InAsSb/InAs: A type-I or a typeII band alignment Phys. Rev. B 5212039 